\title{
Transmission of SARS-CoV-2: an update of current literature
}

\author{
Kishan P. Patel ${ }^{1}$ - Srinivas R. Vunnam ${ }^{1}$ • Puja A. Patel ${ }^{2} \cdot$ Kaleigh L. Krill $^{3} \cdot$ Parker M. Korbitz $^{4} \cdot$ John P. Gallagher $^{4}$. \\ Jane E. Suh ${ }^{5}$ Rama R. Vunnam ${ }^{3}$ (i)
}

Received: 9 May 2020 / Accepted: 19 June 2020 / Published online: 7 July 2020

(C) Springer-Verlag GmbH Germany, part of Springer Nature 2020

\begin{abstract}
Severe acute respiratory syndrome coronavirus 2 (SARS-CoV-2), the etiologic agent for the 2019 coronavirus disease (COVID19) pandemic, has caused a public health emergency. The need for additional research in viral pathogenesis is essential as the number of cases and deaths rise. Understanding the virus and its ability to cause disease has been the main focus of current literature; however, there is much unknown. Studies have revealed new findings related to the full transmission potential of SARS-CoV-2 and its subsequent ability to cause infection by different means. The virus is hypothesized to be of increased virulence compared with previous coronavirus that caused epidemics, in part due to its overall structural integrity and resilience to inactivation. To date, many studies have discussed that the rationale behind its transmission potential is that viral RNA has unexpectedly been detected in multiple bodily fluids, with some samples having remained positive for extended periods of time. Additionally, the receptor by which the virus gains cellular entry, ACE2, has been found to be expressed in different human body systems, thereby potentiating its infection in those locations. In this evidence-based comprehensive review, we discuss various potential routes of transmission of SARS-CoV-2 - respiratory/droplet, indirect, fecal-oral, vertical, sexual, and ocular. Understanding these different routes is important as they pertain to clinical practice, especially in taking preventative measures to mitigate the spread of SARS-CoV-2.
\end{abstract}

Keywords COVID-19 $\cdot$ SARS-CoV-2 $\cdot$ Transmission $\cdot$ Routes $\cdot$ Modes $\cdot$ RNA

\section{Introduction}

SARS-CoV-2, previously known as the 2019 novel coronavirus, is an enveloped non-segmented positive-sense RNA virus responsible for the 2019 coronavirus disease (COVID-19) pandemic. It is classified as a beta coronavirus, rendering

Rama R. Vunnam

rvunnam@pennstatehealth.psu.edu

1 Department of Internal Medicine, University of Nebraska Medical Center, Omaha, NE, USA

2 Nova Southeastern University Kiran C. Patel College of Osteopathic Medicine, Fort Lauderdale, FL, USA

3 Department of Medicine, Division of Hospital Medicine, Penn State Health Milton S. Hershey Medical Center, Hershey, PA 17033, USA

4 University of Nebraska Medical Center College of Medicine, Omaha, NE, USA

5 Department of Gastroenterology and Hepatology, University of Nebraska Medical Center, Omaha, NE, USA mammalian hosts susceptible to infection. Six other coronaviruses have been identified to infect human hosts, resulting in epidemics, including severe acute respiratory syndrome-related coronavirus (SARS-CoV) and Middle East respiratory syndrome coronavirus (MERS-CoV) [1]. As of May 7, 2020, the global count has reached 3,672,238 confirmed cases with 254,045 COVID-19-related deaths [2], causing growing public health concern. $\mathrm{R}_{\mathrm{t}}$, the effective reproduction number, approximates the potential for a virus to spread given a specific measure of time in relation to control measures in place. As suggested by Inglesby in an article in $J A M A$, without measures such as social distancing in place, the $\mathrm{R}_{\mathrm{t}}$ is estimated to be similar to that of its value in January 2020 ranging between 2 and 4 [3].

The transmission potential of the virus may be due in part to its structure and overall tenacity. SARS-CoV-2 was found to have one of the hardest outer protective shells among all coronaviruses. This is believed to result in more stable viral particles, resulting in its greater resilience in bodily fluid [4]. In a NEJM study, SARS-CoV-2 was found to remain viable, in aerosolized form for up to $3 \mathrm{~h}$, and stable on plastic and 
stainless steel surfaces for up to $72 \mathrm{~h} \mathrm{[5]}$. The virus was found to be more stable on smooth surfaces [6]. However, it should be noted that the findings presented are in the absence of in vivo data and based solely on a limited number of studies, potentially creating a gap in our understanding.

Studies have demonstrated that the mechanism by which SARS-CoV-2 gains cellular entry in the respiratory tract is through the angiotensin-converting enzyme 2 (ACE2) receptor [7]. The first step involves viral attachment to ACE2 to enable host cell entry; the second step involves activation of the virus internalization process by proteases, particularly TMPRSS2 [8]. ACE2 has been noted to be expressed in various tissues and organ systems throughout the body, including the central nervous system, gastrointestinal system, heart, lung, testes, and kidney [9-11]. It is hypothesized that this ACE2-mediated mechanism impacts the tropism of SARS-CoV-2 and its ability to invade different organ systems and cause damage $[9,10]$.

Studies have shown the detection of viral RNA in various bodily fluid samples. Wang et al. collected 1070 specimens from 205 patients with COVID-19 disease: bronchoalveolar lavage fluid (14 of 15; 93\%), sputum (72 of 104; 72\%), nasal swabs (5 of $8 ; 63 \%)$, pharyngeal swabs (126 of $398 ; 32 \%)$, feces (44 of 153; 29\%), blood ( 3 of $307 ; 1 \%)$, and urine ( 0 of 72; 0\%) [12]. A preprint study reported a COVID-19 patient with no urinary symptoms having tested positive for SARSCoV-2 in the urine [13]. One case report depicted a patient with meningitis/encephalitis associated with COVID-19, whereby SARS-CoV-2 RNA was detected in cerebrospinal fluid (CSF) [14], whereas another study of pediatric COVID-19 patients showed that four patients with neurological symptoms had negative CSF samples for viral RNA [15]. A prospective study by Sun J et al. examining 49 COVID-19 patients in China noted that persistent prolonged viral RNA shedding has been detected in body fluids, particularly that of nasopharyngeal and fecal samples [16]. Additionally, while viral shedding has been widely noted, a study by Xiao et al. had successfully isolated infectious SARS-CoV-2 from fecal matter [17]. It is important to note that although quantitative viral RNA has been detected from various body sources, the ability to recover viable virus is of critical importance to understand transmission routes. As some of the studies included in this review lack this data, it may be very difficult to draw reasonable conclusions.

In this review, the latest literature pertaining to the potential for various routes of transmission of SARS-CoV-2 will be discussed.

\section{Methods}

This narrative review serves to provide information on the latest literature available pertaining to the transmission potential of SARS-CoV-2. This paper was not systematically conducted, and the data presented was made available according to the authors' preference and may be subject to bias or missing data. As the COVID-19 pandemic is evolving, research becomes progressively available, keeping in mind that some of the studies collected thus far may consist of case reports or series with lowquality methodology as they were obtained when this review was written. Therefore, the conclusions of this review may be limited in evidence as further research becomes readily available.

\section{Routes of transmission}

\section{Respiratory transmission}

Similar to SARS-CoV and MERS-CoV, SARS-CoV-2 is predominantly characterized as a respiratory tract infection. The virus commonly presents with nonspecific lower respiratory tract infection symptoms, such as fever, cough, and shortness of breath [18]. The first cases of COVID-19 took place in Wuhan, China, where a group of individuals were evaluated for pneumonia of unknown etiology [19]. Following the outbreak, it was suspected that the virus propagated by means of community and intrafamilial spread [20]. The transmission is thought to be predominately person to person, either by direct contact or through droplets originating from infected individuals [21]. Droplets can be formed through coughing, sneezing, singing, breathing, and speaking [22]. Droplet transmission involves exposing an entry point, such as mucosa (nose and mouth) or conjunctiva, to potentially infective respiratory droplets (typically $>5-10 \mu \mathrm{m}$ in diameter) produced by someone having respiratory symptoms within a $1 \mathrm{~m}$ proximity [23]. The inhalation of small, exhaled respiratory droplets containing infectious virions is thought to occur; there is a growing concern for long-range human-to-human infection should these droplets remain airborne and viable [24].

Another medium to consider is airborne dust, as inhalation of virus-laden fine particles may transport the virus in deeper bronchial and alveolar regions [24]. A study analyzing COVID-19 patients from three hospitals in China demonstrated higher positive rates of viral RNA in bronchoalveolar lavage fluid, sputum, and nasal swabs [12]. Furthermore, in a study evaluating environmental contamination in symptomatic patients, one of the three subjects had $87 \%$ of his tested room sites, including air outlets, return positive for viral RNA despite only having mild upper respiratory tract involvement. Environmental contamination through airflow may perpetuate viral transmission through infectious droplets [25].

\section{Indirect transmission}

Compared with direct human-to-human spread, the role of indirect transmission is less understood. Airborne transmission occurs when larger respiratory droplets, which have evaporated, or dust particles harbor microbes in the droplet nuclei with a 
size $<5 \mu \mathrm{m}$ in diameter. This allows microbes to remain in the air for longer periods of time and travel greater than $1-\mathrm{m}$ distance. Airborne transmission becomes a threat when aerosolization of particles takes place, especially in essential procedures such as endotracheal intubation, bronchoscopy, and cardiopulmonary resuscitation [23]. Liu et al. investigated the aerodynamic nature of the virus based on findings from hospitals in China. The concentration of viral RNA in aerosols detected in isolation wards and ventilated patient rooms was very low but elevated in toilet areas. Additionally, airborne viral RNA levels in the majority of public areas were undetectable with the exception of a few areas prone to crowding [26].

In addition to aerosolization of microbe particles, infection secondary to contact with contaminated objects or exposure to fomites may occur. A study from the NEJM characterized the viability of the virus in multiple surfaces of inoculated samples, with their results indicating that indirect transmission by means of fomite and aerosolization was plausible [5]. In an early study by Cai et al., COVID-19 patients were tracked to identify their environmental exposures within a shopping mall. The findings suggested that viral spread was unlikely due to respiratory droplet transmission alone. Direct contact with other subjects, asymptomatic individuals, or exposure to contaminated environments accounted for transmission in some subjects [27]. Moriarty et al. highlighted the transmission potential of the virus on cruise ships totaling more than 800 confirmed cases; viral RNA was discovered on multiple surfaces of cabins in symptomatic and asymptomatic individuals despite quarantining efforts, questioning the role of transmission by fomites [28]. Additionally, a preprint study conducted by Santarpia et al. highlights environmental viral shedding observed in 13 quarantined individuals in the USA; of 163 surface and aerosol samples collected, $126(77.3 \%)$ tested positive by reverse transcriptase-polymerase chain reaction (RT-PCR) for SARS-CoV-2. Furthermore, $80.4 \%$ of all room surface samples tested positive for the presence of viral RNA, supporting significant indirect contamination and likely airborne transmission [29]. It should be noted that to the best of our knowledge, no viable virus has been isolated from fomite samples indicating limited data on its transmissibility.

\section{Fecal-oral route of transmission}

Thus far, there have been many studies analyzing the possibility of fecal-oral transmission of COVID-19. Potential SARS-CoV-2 infection in the gastrointestinal tract has been discussed in regard to the expression of ACE2 and TMPRSS2 in the epithelium [10,30]. Additionally, studies have noted that its fecal-oral transmission potential may lie in the fact that prolonged viral shedding can occur in fecal matter-one case reported an asymptomatic COVID-19 patient experiencing viral detection in the stool for up to 42 days, while nasopharyngeal sampling was negative [31]. Prolonged viral detection in stool samples has also been detected in pediatric patients following the recovery of COVID-19 pneumonia [32]. In another study involving pediatric COVID-19 patients postdischarge by Zhang et al., all subjects had positive stool RTPCR results after 10 days, without positive throat swabs, clinical symptoms, or imaging findings [32]. Similar findings were demonstrated in adults, whereby patients had positive RT-PCR results in anal swabs after recovery, yet still met criteria for hospital discharge due to negative nasopharyngeal testing [33].

While prolonged viral shedding in the stool has been noted, whether or not these particles are infectious and have the potential of being spread fecal-orally is questionable. Supporting its potentiation, one study by Zhang et al. has characterized the presence of live virus in stool; they were able to successfully culture SARS-CoV-2 in Vero cells which was isolated from a stool specimen of a patient with severe COVID-19 pneumonia [34]. Additionally, a study published in JAMA by Ong et al. supported the possibility of this transmission by demonstrating extensive environmental contamination from a symptomatic COVID-19 patient. Samples were collected from the room of a patient whose fecal matter tested positive for SARS-CoV-2 by RT-PCR prior to routine cleaning, including from the surface of the toilet bowl, inside of the bowl, and door handle - all of which tested positive. However, samples obtained post-cleaning were negative, implying that current decontamination measures are effective. These findings suggested that viral shedding in the stool could be contributing to a possible route of transmission [25].

Yeo et al. discussed the clinical implications that fecal-oral transmission of COVID-19 may have in infection control, especially in areas with poor sanitation [35]. With new findings, it was recommended that when handling stool of COVID-19 patients, strict precautions be practiced [35]. In fact, the detection of SARS-CoV-2 in untreated wastewater was confirmed in Australia [36]. Yeo et al. also discussed the need for hospital-directed recommendations regarding proper management and disinfection of sewage due to growing concerns for the existence of fecal-oral transmission [35].

\section{Vertical route of transmission}

Although vertical transmission of COVID-19 has been studied, there still remains a need for further conclusive evidence. Certain studies have suggested evidence for vertical transmission on the basis that some neonates born to COVID-19positive mothers had elevated IgM antibodies following birth [37-39]. In a study by Dong et al., a neonate born to a COVID-19-positive mother was found to have elevated IgM antibodies $2 \mathrm{~h}$ after birth but tested negative for COVID-19 on nasopharyngeal specimens. Typically, IgM antibodies do not appear until 3-7 days after infection in part due to its molecular structure, but this elevation was present soon after birth in 
the setting of negative maternal vaginal secretions for SARSCoV-2 $[38,39]$. Another study conducted by Zeng et al. examined 6 pregnant COVID-19 patients and highlighted that two infants had elevated IgM levels [39].

In a study by Parazzini et al., COVID-19 mothers with both vaginal and cesarean deliveries were assessed (6 and 31, respectively). Two neonates tested positive for SARS-CoV-2 on RT-PCR testing; three neonates had elevated SARS-CoV-2 IgG and IgM levels but tested negative on RT-PCR. It was concluded that the rate of vertical or peripartum transmission of COVID-19 is low to none for cesarean delivery, but no data was available for vaginal delivery [40]. Additionally, a study involving 31 COVID-19 pregnant mothers reported no vertical transmission in their neonates or placentas [41].

Zamaniyan et al. reported a pregnant woman with severe COVID-19 pneumonia having given birth to a preterm baby at 32 weeks gestation with no evidence of SARS-CoV-2 infection. However, testing for COVID-19 by RT-PCR was positive in both an amniotic sample and a second nasal and throat test the neonate underwent $24 \mathrm{~h}$ after birth via cesarean delivery; testing was negative in the vaginal secretion sample, umbilical cord blood, and first neonate test. Since the amniotic fluid and the neonate tested positive, it may suggest that the newborn was affected intrauterine by SARS-CoV-2 [42]. To oppose, in a retrospective review of nine COVID-19 pregnant mothers who underwent cesarean section, six patients had samples of amniotic fluid, cord blood, neonatal throat swab, and breastmilk samples tested for SARS-CoV-2, and all were negative [43]. Furthermore, there has been a case of neonate in China who tested positive for COVID-19 via RT-PCR of pharyngeal swabs $36 \mathrm{~h}$ after birth; however, vertical transmission in the case was not confirmed [44].

The impact that COVID-19 pregnancies can have on neonates is largely unknown. In a clinical analysis of 10 neonates born from nine COVID-19 mothers by Zhu et al., it was found that perinatal infection of SARS-CoV-2 may lead to adverse effects in newborns, as some of the neonates experienced fetal distress, premature labor, respiratory distress, thrombocytopenia, abnormal liver function tests, and even death [45]. A recent study in JAMA by Baud et al. depicted a case of miscarriage during the second trimester in a COVID-19 female related to placental infection with SARS-CoV-2. The fetal surface of the placenta tested positive for SARS-CoV-2; histopathology revealed mixed inflammatory infiltrates and funisitis. Although vertical transmission was not proven, no other etiology of fetal demise was identified [46]. Nevertheless, further research is needed to determine whether SARS-CoV-2 crosses the placental barrier.

\section{Sexual route of transmission}

In a cross-sectional study examining 34 adult males recovering from COVID-19, SARS-CoV-2 was not detected in any of the subjects' semen approximately 1 month after initial disease confirmation. However, the findings were unable to definitively rule out the presence of SARS-CoV-2 in seminal fluid due to a lack of testing during the acute phase of infection [47]. However, a recent cohort study by Li et al. demonstrated that semen testing for SARS-CoV-2 resulted positive for six of the 38 male COVID-19 patients, four of which were in the acute stage of infection and two being in the recovery phase [48]. The study by Pan et al. also characterized that there was low overlapping gene expression of ACE2 and TMPRSS2 in human testes, suggesting that the SARS-CoV-2 would not likely be able to gain testicular cellular entry via this mechanism [47]. However, as stated by Sama et al., ACE2 has been noted to be highly expressed in the testis [11].

To et al. noted the detection of SARS-CoV-2 in selfcollected saliva specimens in $91.7 \%$ (11/12) of COVID-19 patients [49]. The need for safe sexual intercourse behavior may be heightened due to the fact that the virus has been detected in high concentrations in saliva and nasal mucosa and may have potential receptor binding in rectal and anal epithelial cells.

\section{Ocular route of transmission}

Lastly, the potential for an ocular route of transmission has recently been explored. Certain studies have noted the detection of viral RNA in conjunctival specimens, while others demonstrated limited evidence of its presence. A case study of an ocular manifestation of COVID-19 presenting as bilateral conjunctivitis 13 days after onset of illness suggested that viral shedding occurs in the eyes; RT-PCR of conjunctival samples on days 14 and 17 following the onset of symptoms tested positive. The conjunctiva was confirmed to be a site of viral replication when he tested positive via RT-PCR of consecutive conjunctival swabs. On day 19 , the conjunctival swab specimen resulted negative [50]. Moreover, 38 COVID-19 patients at a hospital center in China were retrospectively reviewed for ocular manifestations; the study concluded that about one-third of patients with COVID-19 had ocular abnormalities, which frequently occurred in patients with more severe disease [51]. In a case series of 38 COVID-19 patients published in JAMA Ophthalmology, 31.6\% (95\% CI, 17.548.7) of subjects had ocular manifestations consistent with conjunctivitis such as conjunctival hyperemia, chemosis, or increased secretions. Similar to the prior study, this more commonly occurred in patients with severe systemic manifestations or abnormal laboratory tests. There was a low prevalence of viral nucleotides in conjunctival specimens of 5.2\% (95\% CI, 0.6-17.8) [51].

A recent case report of the first patient in Italy to be diagnosed with COVID-19 depicted bilateral conjunctivitis, in addition to fever, respiratory symptoms, nausea, and vomiting. SARS-CoV-2 RNA was detected via conjunctival 
swab on day 3 of hospitalization while still having persistent conjunctivitis - it remained positive until day 21 and again on day 27 (conjunctivitis resolved on day 20). In order to demonstrate that the ocular samples contained infectious virus, an RNA-positive ocular sample was inoculated in Vero E6 cells, with a cytopathic effect being observed 5 days later. Viral replication was confirmed via RT-PCR from purified RNA in the cell growth medium. Implications of these findings are that ocular fluid of COVID-19 patients may contain infectious virus and serve as a possible source of infection $[52,53]$.

However, a few studies have demonstrated limited evidence for an ocular route of transmission. In a study of 17 COVID-19 patients, tears were sampled and all samples resulted negative for the presence of SARS-CoV-2, while nasopharyngeal samples were positive. Likewise, patients with upper respiratory tract infections did not demonstrate any evidence of viral shedding in tears, suggesting that transmission through tears is likely low [54]. A couple of preprint studies have arrived at similar conclusions. In a study by Sun et al. involving 72 patients with laboratory-confirmed COVID-19, only two patients had conjunctivitis, but SARS-CoV-2 RNA fragments were found in the ocular discharge of one of the two [55]. Another preprint study which was a retrospective cohort study enrolled 67 confirmed and suspected COVID-19 patients, of which 63 were laboratory confirmed. Of the 63 laboratory-confirmed patients, conjunctival specimens from one patient were positive on PCR testing and two patients had probable positive results - none of these three had ocular symptoms [56].

\section{Conclusion}

The outbreak of COVID-19 and evolving pandemic warrant the need for better infection control by means of identifying viral sources. Emerging studies have detected the presence of viral RNA in multiple regions of the human body, raising concern for its ease of transmissibility. Thus far, COVID-19 was originally thought to be spread by respiratory droplets; however, newer studies have highlighted the potential for alternative routes. Detection of viral RNA shedding in multiple bodily fluids/samples suggests the potential for additional modes of transmission, such as bloodborne, urinary, and fecal-respiratory. Transmission by such means remains controversial given the limited supporting data for each mode. Clinicians should be aware that the detection of viral RNA in numerous bodily fluids can potentiate new routes of transmission as more data on SARS-CoV-2 emerges becoming more readily available.

These findings emphasize the importance of control measures as they could impact daily practices, from precautions taken by the general public to prevent asymptomatic spread of disease to the isolation precautions practiced in healthcare facilities. Limitations to consider when interpreting certain studies discussing transmission routes are availability of research, sample size of patients, and testing capacity in relation to diagnostic supply shortages. The role of whole genome sequencing in characterizing the presence of SARS-CoV-2 has been discussed as a comprehensive detection method. The limitations with this method include cost, time, and complications; however, it may play a part in elucidating further understanding of viral transmission pathways. As COVID-19 is not completely understood, the need for further research confirming and/or refuting these potential routes of transmission remains a priority.

Authors' contributions The authors contributed to and reviewed all aspects of the article.

\section{Compliance with ethical standards}

Competing interests The authors declare that they have no competing interests.

\section{References}

1. Guo Y-R, Cao Q-D, Hong Z-S et al (2020) The origin, transmission and clinical therapies on coronavirus disease 2019 (COVID-19) outbreak - an update on the status. Military Med Res 7(1):11. https://doi.org/10.1186/s40779-020-00240-0

2. World Health Organization. (2020) Coronavirus disease 2019 (Covid-19) situation report - 108

3. Inglesby TV (2020) Public health measures and the reproduction number of SARS-CoV-2. JAMA 323(21):2186-2187. https://doi. org/10.1001/jama.2020.7878

4. Goh GK-M, Dunker AK, Foster JA et al (2020) Shell disorder analysis predicts greater resilience of the SARS-CoV-2 (COVID19) outside the body and in body fluids. Microb Pathog 144: 104177. https://doi.org/10.1016/j.micpath.2020.104177

5. van Doremalen N, Bushmaker T, Morris DH et al (2020) Aerosol and surface stability of SARS-CoV-2 as compared with SARSCoV-1. N Engl J Med 382(16):1564-1567. https://doi.org/10. 1056/NEJMc2004973

6. Chin AWH, Chu JTS, Perera MRA et al Stability of SARS-CoV-2 in different environmental conditions. Lancet Microbe. https://doi. org/10.1016/S2666-5247(20)30003-3

7. Liu Z, Xiao X, Wei X et al (2020) Composition and divergence of coronavirus spike proteins and host ACE2 receptors predict potential intermediate hosts of SARS-CoV-2. J Med Virol 92(6):595601. https://doi.org/10.1002/jmv.25726

8. Stopsack KH, Mucci LA, Antonarakis ES et al (2020) TMPRSS2 and COVID-19: serendipity or opportunity for intervention? Cancer Discov. https://doi.org/10.1158/2159-8290.Cd-20-0451

9. Baig AM, Khaleeq A, Ali U et al (2020) Evidence of the COVID19 virus targeting the CNS: tissue distribution, host-virus interaction, and proposed neurotropic mechanisms. ACS Chem Neurosci 11(7):995-998. https://doi.org/10.1021/acschemneuro.0c00122

10. Zhang H, Li HB, Lyu JR et al (2020) Specific ACE2 expression in small intestinal enterocytes may cause gastrointestinal symptoms and injury after 2019-nCoV infection. Int J Infect Dis. https://doi. org/10.1016/j.jijid.2020.04.027 
11. Sama IE et al (2020) Circulating plasma concentrations of angiotensin-converting enzyme 2 in men and women with heart failure and effects of renin-angiotensin-aldosterone inhibitors. Eur Heart J 41(19):1810-1817. https://doi.org/10.1093/eurheartj/ ehaa373

12. Wang W, Xu Y, Gao R et al (2020) Detection of SARS-CoV-2 in different types of clinical specimens. JAMA. https://doi.org/10. 1001/jama.2020.3786

13. Peng, L., Liu, J., Xu, W., et al. (2020). 2019 novel coronavirus can be detected in urine, blood, anal swabs and oropharyngeal swabs samples. medRxiv, 2020.2002.2021.20026179. https://doi.org/10. 1101/2020.02.21.20026179

14. Moriguchi T, Harii N, Goto J et al (2020) A first case of meningitis/ encephalitis associated with SARS-Coronavirus-2. Int J Infect Dis 94:55-58. https://doi.org/10.1016/j.ijid.2020.03.062

15. Nathan N, Prevost B, Corvol HAtypical presentation of COVID-19 in young infants. Lancet. https://doi.org/10.1016/S0140-6736(20) 30980-6

16. Jiufeng S, Jianpeng X, Ruilin S, Xi T, Chumin L, Huifang L, ... Changwen K (2020) Prolonged persistence of SARS-CoV-2 RNA in body fluids. Emerg Infect Dis J, 26(8). https://doi.org/10.3201/ eid2608.201097

17. Fei X, Jing S, Yonghao X et al (2020) Infectious SARS-CoV-2 in feces of patient with severe COVID-19. Emerg Infect Dis J 26(8). https://doi.org/10.3201/eid2608.200681

18. Wu Y-C, Chen C-S, Chan Y-J (2020) The outbreak of COVID-19: an overview. J Chin Med Assoc 83(3):217-220. https://doi.org/10. 1097/JCMA.0000000000000270

19. Wu Z, McGoogan JM (2020) Characteristics of and important lessons from the coronavirus disease 2019 (COVID-19) outbreak in China: summary of a report of 72314 cases from the Chinese Center for Disease Control and Prevention. JAMA 323(13):12391242. https://doi.org/10.1001/jama.2020.2648

20. Jiaye L, Xuejiao L, Shen Q et al (2020) Community transmission of severe acute respiratory syndrome coronavirus 2, Shenzhen, China, 2020. Emerg Infect Dis J 26(6). https://doi.org/10.3201/eid2606. 200239

21. Rothan HA, Byrareddy SN (2020) The epidemiology and pathogenesis of coronavirus disease (COVID-19) outbreak. J Autoimmun 109:102433. https://doi.org/10.1016/j.jaut.2020. 102433

22. Anderson EL, Turnham P, Griffin JR, Clarke CC (2020) Consideration of the aerosol transmission for COVID-19 and public health. Risk Anal 40(5):902-907. https://doi.org/10.1111/risa. 13500

23. Modes of transmission of virus causing COVID-19: implications for IPC precaution recommendations: scientific brief, 27 March 2020. Retrieved from https://apps.who.int/iris/handle/ $10665 / 331601$

24. Qu G, Li X, Hu L et al (2020) An imperative need for research on the role of environmental factors in transmission of novel coronavirus (COVID-19). Environ Sci Technol 54(7):3730-3732. https:// doi.org/10.1021/acs.est.0c01102

25. Ong SWX, Tan YK, Chia PY et al (2020) Air, surface environmental, and personal protective equipment contamination by severe acute respiratory syndrome coronavirus 2 (SARS-CoV-2) from a symptomatic patient. JAMA 323(16):1610-1612. https://doi.org/ 10.1001/jama.2020.3227

26. Liu Y, Ning Z, Chen Y et al (2020) Aerodynamic analysis of SARS-CoV-2 in two Wuhan hospitals. Nature. https://doi.org/10. 1038/s41586-020-2271-3

27. Cai J, Sun W, Huang J et al (2020) Indirect virus transmission in cluster of COVID-19 cases, Wenzhou, China, 2020. Emerg Infect Dis 26(6). https://doi.org/10.3201/eid2606.200412

28. Moriarty LF, Plucinski MM, Marston BJ, et al. Public health responses to COVID-19 outbreaks on cruise ships — worldwide,
February-March 2020. MMWR Morb Mortal Wkly Rep 2020;69:347-352. https://doi.org/10.15585/mmwr.mm6912e3

29. Santarpia, J. L., Rivera, D. N., Herrera, V., et al. (2020). Transmission potential of SARS-CoV-2 in viral shedding observed at the University of Nebraska Medical Center. medRxiv, 2020.2003.2023.20039446. doi:https://doi.org/10.1101/2020.03. 23.20039446

30. Iwata-Yoshikawa N, Okamura T, Shimizu Y et al (2019) TMPRSS2 contributes to virus spread and immunopathology in the airways of murine models after coronavirus infection. J Virol 93(6):e01815-e01818. https://doi.org/10.1128/JVI.01815-18

31. Jiang X, Luo M, Zou Z et al (2020) Asymptomatic SARS-CoV-2 infected case with viral detection positive in stool but negative in nasopharyngeal samples lasts for 42 days. J Med Virol. https://doi. org/10.1002/jmv. 25941

32. Zhang T, Cui $X$, Zhao $X$ et al (2020) Detectable SARS-CoV-2 viral RNA in feces of three children during recovery period of COVID19 pneumonia. J Med Virol. https://doi.org/10.1002/jmv.25795

33. Liu J, Xiao Y, Shen Y et al (2020) Detection of SARS-CoV-2 by RT-PCR in anal from patients who have recovered from coronavirus disease 2019. J Med Virol. https://doi.org/10.1002/jmv.25875

34. Zhang Y, Chen C, Zhu S et al (2020) Isolation of 2019-nCoV from a stool specimen of a laboratory-confirmed case of the coronavirus disease 2019 (COVID-19)[J]. China CDC Weekly 2(8):123-124. https://doi.org/10.46234/ccdcw2020.033

35. Yeo C, Kaushal S, Yeo D (2020) Enteric involvement of coronaviruses: is faecal-oral transmission of SARS-CoV-2 possible? Lancet Gastroenterol Hepatol 5(4):335-337. https://doi.org/ 10.1016/S2468-1253(20)30048-0

36. Ahmed W, Angel N, Edson J et al (2020) First confirmed detection of SARS-CoV-2 in untreated wastewater in Australia: a proof of concept for the wastewater surveillance of COVID-19 in the community. Sci Total Environ 728:138764. https://doi.org/10.1016/j. scitotenv.2020.138764

37. Kimberlin DW, Stagno S (2020) Can SARS-CoV-2 infection be acquired in utero?: more definitive evidence is needed. JAMA. https://doi.org/10.1001/jama.2020.4868

38. Dong L, Tian J, He S et al (2020) Possible vertical transmission of SARS-CoV-2 from an infected mother to her newborn. JAMA. https://doi.org/10.1001/jama.2020.4621

39. Zeng H, Xu C, Fan J et al (2020) Antibodies in infants born to mothers with COVID-19 pneumonia. JAMA. https://doi.org/10. 1001/jama.2020.4861

40. Parazzini F, Bortolus R, Mauri PA et al (2020) Delivery in pregnant women infected with SARS-CoV-2: a fast review. Int J Gynaecol Obstet. https://doi.org/10.1002/ijgo.13166

41. Karimi-Zarchi, M., Neamatzadeh, H., Dastgheib, S. A., et al. (2020). Vertical transmission of coronavirus disease 19 (COVID19) from infected pregnant mothers to neonates: a review. Fetal Pediatr Pathol, 1-5. https://doi.org/10.1080/15513815.2020. 1747120

42. Zamaniyan M, Ebadi A, Mir SA et al (2020) Preterm delivery in pregnant woman with critical COVID -19 pneumonia and vertical transmission. Prenat Diagn. https://doi.org/10.1002/pd.5713

43. Chen H, Guo J, Wang C et al (2020) Clinical characteristics and intrauterine vertical transmission potential of COVID-19 infection in nine pregnant women: a retrospective review of medical records. Lancet 395(10226):809-815. https://doi.org/10.1016/s01406736(20)30360-3

44. Wang S, Guo L, Chen L et al (2020) A case report of neonatal COVID-19 infection in China. Clin Infect Dis. https://doi.org/10. 1093/cid/ciaa225

45. Zhu H, Wang L, Fang C et al (2020) Clinical analysis of 10 neonates born to mothers with 2019-nCoV pneumonia. Transl Pediatr 9(1):51-60. https://doi.org/10.21037/tp.2020.02.06 
46. Baud D, Greub G, Favre G et al (2020) Second-trimester miscarriage in a pregnant woman with SARS-CoV-2 infection. JAMA. https://doi.org/10.1001/jama.2020.7233

47. Pan F, Xiao X, Guo J et al (2020) No evidence of SARS-CoV-2 in semen of males recovering from COVID-19. Fertil Steril. https:// doi.org/10.1016/j.fertnstert.2020.04.024

48. Li D, Jin M, Bao P, Zhao W, Zhang S (2020) Clinical characteristics and results of semen tests among men with coronavirus disease 2019. JAMA Netw Open 3(5):e208292-e208292. https://doi.org/ 10.1001/jamanetworkopen.2020.8292

49. To, K. K.-W., Tsang, O. T.-Y., Chik-Yan Yip, C., et al. (2020). Consistent detection of 2019 novel coronavirus in saliva. Clinical infectious Dis ciaa149. https://doi.org/10.1093/cid/ciaa149

50. Chen, L., Liu, M., Zhang, Z., et al. (2020). Ocular manifestations of a hospitalised patient with confirmed 2019 novel coronavirus disease. Br J Ophthalmol, bjophthalmol-2020-316304. https://doi.org/ 10.1136/bjophthalmol-2020-316304

51. Wu P, Duan F, Luo C et al (2020) Characteristics of ocular findings of patients with coronavirus disease 2019 (COVID-19) in Hubei Province, China. JAMA Ophthalmol. https://doi.org/10.1001/ jamaophthalmol.2020.1291
52. Colavita F, Lapa D, Carletti F et al (2020) SARS-CoV-2 isolation from ocular secretions of a patient with COVID-19 in Italy with prolonged viral RNA detection. Ann Intern Med. https://doi.org/10. 7326/m20-1176

53. Important coronavirus updates for ophthalmologists. (2020). Retrieved from https://www.aao.org/headline/alert-importantcoronavirus-context

54. Seah IYJ, Anderson DE, Kang AEZ et al (2020) Assessing viral shedding and infectivity of tears in coronavirus disease 2019 (COVID-19) patients. Ophthalmology. https://doi.org/10.1016/j. ophtha.2020.03.026

55. Sun, X., Zhang, X., Chen, X., et al. (2020). The infection evidence of SARS-COV-2 in ocular surface: a single-center cross-sectional study. medRxiv, doi:https://doi.org/10.1101/2020.02.26.20027938

56. Zhou, Y., Zeng, Y., Tong, Y., \& Chen, C. (2020). Ophthalmologic evidence against the interpersonal transmission of 2019 novel coronavirus through conjunctiva. medRxiv, doi:https://doi.org/10. $1101 / 2020.02 .11 .20021956$

Publisher's note Springer Nature remains neutral with regard to jurisdictional claims in published maps and institutional affiliations. 